\title{
Um Relato de Experiência: Ensinando Robótica por Meio de Microcontroladores em uma Escola Profissional de Ensino Médio
}

\author{
Mateus Lima Alves, José Robertty de Freitas Costa, Paulo Miranda e Silva Sousa \\ Paulo Ricardo da Silva Lopes, Carla Ilane Moreira Bezerra \\ ${ }^{1}$ Campus de Quixadá - Universidade Federal do Ceará (UFC) \\ 63.902-580 - Quixadá - CE - Brasil \\ \{mateuslima.ti, robertty, paulomirandamss, prslopes1\}@alu.ufc.br \\ carlailaneeufc.br
}

\begin{abstract}
The enthusiasm for robotics and automation encourages many young people to participate in projects involving the construction of hardware and software. Microcontrollers are a "backbone" of robotics and automation projects. Such devices control and manage the other system components. For this, it is necessary to know in programming and programming, to become fundamental to the young developer. In this context, a proposed proposal was proposed as an initiative for the study of robotics. An action visa is part of the high school in the area of robotics, through a course of microcontrollers. This article aims to make an account of the extension action, with its planning, execution, results achieved and future work.
\end{abstract}

Resumo. $O$ entusiasmo pela robótica e automação incentiva vários jovens a participarem de projetos que envolvem a construção de hardware e software. Os microcontroladores são a "espinha dorsal" de projetos de robótica e automação. Tais dispositivos controlam e gerenciam os demais componentes do sistema. Para isso, conhecimentos em eletrônica e programação, tornam-se fundamentais para o jovem desenvolvedor. Nesse contexto, uma ação de extensão foi proposta, como uma iniciativa ao estudo de robótica. A ação visa inserir alunos do ensino médio à área da robótica, por meio de um curso de microcontroladores. $O$ presente artigo tem como objetivo fazer um relato da ação de extensão, apresentando seu planejamento, execução, resultados alcançados e trabalhos futuros.

\section{Introdução}

Atualmente, os estudantes do ensino básico estão imersos em um ambiente em que a tecnologia é facilmente percebida: carros, celulares e computadores são exemplos que todos conhecem e muitos utilizam, no entanto, poucos entendem dessas tecnologias [Benitti et al. 2009]. Esse tipo de tecnologia é composto por componentes físicos, denominados hardware, que são coordenados por softwares que gerenciam esses recursos físicos. A união desses dois eixos gera um sistema automatizado e a compressão desse sistema compõe a robótica [Barrientos and Barrientos 2007].

A robótica é uma área relativamente recente que tem ganhado espaço no mercado. Essa área se envolve de maneira intrínseca, com outras grandes áreas como a eletrônica, 
computação e mecânica. Esse domínio trata do estudo e desenvolvimento de sistemas automáticos e controlados por chips de circuitos integrados [Miyagi and Villani 2008].

No processo de ensino aprendizado, o aluno passa para a posição ativa quando abstrai um problema do cotidiano e compreende os mecanismos que o rege. A utilização da tecnologia nesse processo está apresentando resultados positivos em países da Europa e América do Norte. Nesses países, o ensino de programação e robótica é aplicado a problemas práticos, resulta na melhoria do nível de abstração de tecnologias e motiva jovens estudantes inseridos em vários contextos [Avila et al. 2016].

$\mathrm{Na}$ literatura brasileira existem vários relatos do ensino de robótica [Nesi et al. 2014, de Jesus and Cristaldo 2014, Neto et al. 2015]. Em um destes relatos, os autores defendem o ensino de robótica na grade curricular do ensino médio, como estímulo aos alunos seguirem a área de exatas [Nesi et al. 2014]. No entanto, o ensino de robótica exige equipamentos de hardware que podem ter alto custo. Dessa forma, o uso de microcontroladores, que são equipamentos de baixo custo, torna-se uma alternativa viável para o ensino de robótica em escolas de ensino médio púbicas.

Nesse contexto, o presente artigo apresenta um relato de experiência de uma atividade de extensão de ensino de robótica no ensino médio utilizando microcontroladores. O projeto foi idealizado pelo Programa de Educação Tutorial - Tecnologia de Informação Conexões de Saberes do Campus da Universidade Federal do Ceará (UFC) em Quixadá, e foi iniciado no ano de 2017 tendo como objetivo permitir a troca de experiências e conhecimentos entre estudantes de Engenharia de Computação e estudantes do Ensino Médio de uma escola profissional da região. O objetivo do projeto é inserir os estudantes do Ensino Médio na área da robótica incentivando-os a desenvolver projetos na área por meio de microcontroladores, que são dispositivos de controle, programáveis e de baixo custo. A abordagem de robótica com microcontroladores utilizada foi down-top, para que os alunos aprendam desde aplicações básicas como acender de um LED àquelas mais completas como a montagem do primeiro robô, um carro de controle remoto.

O texto está organizado da seguinte forma. A Seção 2 descreve a fundamentação teórica que embasou o projeto, contendo a base teórica do ensino de robótica e conceitos relacionados à utilização de microcontroladores. A metodologia aplicada no trabalho, o levantamento de demanda e a solução prática para o problema estão dispostos na Seção 3. O desenvolvimento da atividade está especificado na Seção 4. Os resultados do projeto são descritos na Seção 5. Por fim, na Seção 6, são apresentadas as conclusões e trabalhos futuros.

\section{Fundamentação Teórica}

Nesta Seção, são detalhados conceitos e trabalhos relacionados ao ensino de robótica e conceitos sobre microcontroladores utilizados na ação de extensão relatada.

\subsection{Ensino de Robótica}

$\mathrm{Na}$ literatura existem vários trabalhos relacionados ao ensino de robótica [Nesi et al. 2014, de Jesus and Cristaldo 2014, Neto et al. 2015].

O trabalho de [Nesi et al. 2014] propõe o ensino e a inclusão da robótica na grade curricular do ensino médio brasileiro, como um estímulo para que jovens estudantes ingressem e permaneçam em cursos de ciências exatas, engenharia e tecnologia. A proposta 
de [Nesi et al. 2014] se baseia em iniciativas na Inglaterra e Austrália, que incluíram em suas grades curriculares obrigatórias disciplinas voltadas a programação e raciocínio lógico. Nesse trabalho, foi desenvolvido uma proposta de plano de aula, cujo objetivo é o desenvolvimento do raciocínio lógico, bem como das características básicas de hardware e software. O projeto de [Nesi et al. 2014] foi a base para o planejamento e desenvolvimento do presente trabalho, pois através dele percebe-se a necessidade do ensino de robótica no âmbito nacional, considerando algumas diretrizes internacionais.

Os autores [de Jesus and Cristaldo 2014] mostram a eficiência do ensino de robótica no processo de aprendizagem, através de uma aplicação de uma olimpíada de robótica envolvendo estudantes do Ensino Médio Integrado de Informática. O trabalho avaliou o desempenho escolar dos alunos antes e durante o projeto, para relacionar o ensino de robótica com o desempenho escolar dos estudantes. Os resultados do trabalho mostram que houve um aumento de $8 \%$ das notas acima de 9,0 da turma selecionada. Além disso, foi avaliada a qualidade do projeto, o resultado foi expressivo, mais de $80 \%$ dos alunos avaliaram o projeto como excelente. Os resultados de [de Jesus and Cristaldo 2014] foram fundamentais para justificar a implementação do presente projeto, pois apresentam uma grande porcentagem de aceitação do projeto por parte dos alunos do Ensino Médio Integrado.

[Neto et al. 2015] coletaram dados de artigos relacionados ao uso da robótica na educação, publicados no Simpósio Brasileiro de Informática na Educação (SBIE), no Workshop de Informática na Educação (WEI) e no Workshop de Robótica na Educação (WRE) no período de 2004 a 2014. Para essa revisão, foram selecionados 65 artigos. Os resultados desse trabalho mostram que grande parte das pesquisas estava localizada nas regiões Sudeste e Nordeste do Brasil. Em um dos questionamentos do trabalho, notou-se que há uma escassez de experimentações educacionais na área, com isso os autores concluem com a afirmação que o ensino da robótica no país ainda lida com muitas propostas, tornando ele uma ciência, até esse tempo, teórica. A revisão sistemática de [Neto et al. 2015] mostrou dados sobre o estado da arte do uso da robótica na educação brasileira, por meio de alguns desses dados percebeu-se a necessidade da utilização da robótica educacional em caráter experimental.

Há diversas iniciativas de levar a robótica para o ambiente escolar, porém com kits completos de desenvolvimento ou plataformas já implementadas, como apresentam os trabalhos de [Benitti et al. 2009] e [Santos et al. 2010].

O trabalho de [Benitti et al. 2009] apresenta um experimento realizado com alunos do primeiro ano do ensino médio. Nesse experimento, os alunos programam robôs para realizarem rotas sobre o mapa de Santa Catarina, aplicando conceitos relacionados à matemática, geografia e programação de computadores.

[Santos et al. 2010] abordam o desenvolvimento de um kit de robótica de baixo custo, levando em consideração o alto custo de kits proprietários já existentes, e utilização do kit nos cursos técnicos e tecnológicos. Os kits desenvolvidos nesse trabalho custaram em torno de 30 reais, mostrando assim a viabilidade do kit para escolas públicas, por exemplo.

Diferente da ação apresentada no trabalho de [Benitti et al. 2009] e [Santos et al. 2010], o curso de microcontroladores possui um custo menor, pois os 
microcontroladores são circuitos integrados compactos, reduzindo assim o custo e o tamanho do produto desenvolvido. Com relação à iniciativa apresentada no artigo de [Santos et al. 2010], o curso de microcontroladores se diferencia no tipo de produto desenvolvido, pois o trabalho de [Santos et al. 2010] mostra o desenvolvimento de kits de robótica educacional, enquanto o curso de microcontroladores propõe a inserção dos alunos de ensino médio por meio de experimentos com microcontroladores.

\subsection{Conceitos de Microcontroladores}

De acordo com [Martins 2005], os microcontroladores estão presentes na grande maioria dos dispositivos eletrônicos atuais, tornando tais dispositivos compactos, o que facilita a manutenção e gerenciamento das funcionalidades internas desses aparelhos.

Os microcontroladores são circuitos integrados, encapsulados em pastilhas, que possuem processador, pinos de entrada e saída e memória. As variações de microcontroladores disponíveis no mercado, de acordo com [Martins 2005], diferem na quantidade de memória interna, velocidade do processamento de dados, quantidade de pinos de entrada e saída, forma de alimentação, consumo de energia, quantidade de periféricos, arquitetura e conjunto de instruções.

Um sistema com diversas funcionalidades necessitaria de um grande número de componentes, porém [Martins 2005] relata em seu trabalho que o mesmo sistema pode ser simplificado ao ser construído com um microcontrolador e conclui que aprender a programar para um microcontrolador é "aprender a resumir circuitos em um único componente".

Vários conceitos de eletrônica estão envolvidos na experimentação em robótica e, dependendo da aplicação, vários circuitos devem ser construídos. Nesse contexto, os microcontroladores são essenciais na síntese desses circuitos. A abordagem da programação para microcontroladores remete o estudante a essa condensação de circuitos em uma única pastilha microcontrolada.

No trabalho de [Ordoñez et al. 2005], é apresentado o PIC16F6281] da Microchip?2. $\mathrm{O}$ autor cita o chip como bem difundido e presente em projetos de automação nos mercados brasileiro e mundial. Além disso, ele relata que a alta performasse do chip o tornara um sucesso de vendas para sistemas embarcados.

Diferentemente do trabalho de [Ordoñez et al. 2005], o presente projeto utiliza o microcontrolador PIC16F870, também da Microchip, que por sua vez é um circuito integrado (CI) com um maior número de módulos que o PIC16F628, porém com a mesma quantidade de instruções (35). A utilização desse dispositivo fundamentou-se por seu baixo custo e sua aplicabilidade em diversos projetos do mercado.

\section{Procedimentos Metodológicos}

Devido o caráter prático e as aplicações pedagógicas deste projeto, a metodologia utilizada neste trabalho foi baseada na Investigação-Ação (IA). De acordo com [Coutinho et al. 2009], a principal característica da metodologia IA é o fato de ser uma

\footnotetext{
${ }^{1}$ http://ww1.microchip.com/downloads/en/DeviceDoc/30569C.pdf

${ }^{2}$ www.microchip.com
} 
pesquisa de essência prática e aplicada, e que se justifica pela necessidade de resolver problemas reais.

Primeiramente foi realizado um levantamento de demanda com a coordenação do curso de Informática da Escola Estadual de Educação Profissional Maria Cavalcante Costa. O levantamento foi realizado por meio de uma reunião, em que se encontravam o diretor da instituição e o coordenador do curso.

Após o levantamento da demanda com os responsáveis pelo curso técnico na escola, viu-se a necessidade de uma ação que incentivasse o interesse dos alunos pela programação e deu-se inicio a elaboração de um curso que aplicasse a programação no desenvolvimento de sistemas microcontrolados com enfoque na robótica.

Em seguida, foi selecionada a turma de segundo ano de informática da escola para a implementação do projeto. O critério de escolha foi os conhecimentos básicos de programação que a turma possuía, mais especificamente em lógica de programação.

Assim como no trabalho de [Nesi et al. 2014], foi desenvolvido um plano de aulas. O esboço foi elaborado baseado na disciplina de Microcontroladores ofertada no curso de Engenharia de Computação da Universidade Federal do Ceará - Campus de Quixadá. As aulas foram planejadas de modo que os alunos imergissem na experimentação da robótica [Neto et al. 2015]. A partir disso, foram estabelecidos os principais pontos a serem abordados no curso: introdução aos paradigmas de programação na linguagem $\mathrm{C}$, configuração e programação de periféricos IO, configuração e programação para o display LCD, introdução a comunicação serial com o módulo USART, introdução ao tratamento de sinais analógicos com o módulo ADC, introdução ao controle de servomotores com módulo PWM e a construção do primeiro robô. Os temas foram definidos por serem conceitos de microcontroladores utilizados na robótica.

No decorrer do curso, foram programadas atividades práticas em grupos onde os alunos tiveram contato com programação e eletrônica na resolução de problemas reais, seguindo a metodologia IA. A organização dos tópicos do curso foi idealizada de modo que ao final do projeto os alunos estejam aptos a construir seu primeiro robô, um carro de controle remoto.

Para as aulas, foram utilizados microcontroladores PIC16F870 e o Ambiente de Desenvolvimento Integrado (IDE) MPLAB X ${ }^{3}$, ambos da empresa Microchip. O microcontrolador escolhido é de baixo custo, de fácil aquisição e bastante utilizado em aplicações de baixa complexidade e a IDE é disponibilizada gratuitamente pela empresa fabricante do chip.

Foram desenvolvidos apresentações de slides e listas de exercícios para que os alunos também compreendessem o conteúdo teórico, todos os materiais foram disponibilizados em um grupo da rede social WhatsApp, em que participaram 25 dos 30 alunos participantes.

\section{Execução do Curso de Robótica}

A primeira turma do curso foi iniciada em maio de 2017 com a participação de 30 alunos e foi planejada para ter 32 horas de duração distribuídas em 2 horas semanais. Após

\footnotetext{
${ }^{3}$ http://www.microchip.com/mplab/mplab-x-ide
} 
a conclusão da primeira turma, houveram algumas modificações nos tópicos do curso conforme a necessidade dos alunos. A segunda turma foi iniciada em março de $2018 \mathrm{e}$ contou com a participação de 40 alunos.

\subsection{Alterações dos Tópicos Entre a Primeira e Segunda Turma}

A principal dificuldade observada na aplicação do curso para a turma de 2017 foi nos paradigmas de programação em $\mathrm{C}$, mesmo após a disciplina de lógica de programação em $\mathrm{C}$. Essa problemática levou os organizadores da ação a adicionarem uma aula de introdução aos paradigmas de programação em $\mathrm{C}$, para que esse obstaculo fosse sanado.

A partir da turma de 2018, foi utilizado o software Open Broadcaster Software 4 (OBS) durante as aulas. O OBS é um software livre para gravação de vídeos, para que as aulas pudessem ser disponibilizadas para os alunos. As aulas gravadas foram publicadas no canal do YouTube do grupo PET Tecnologia da Informação Conexões de Saberes, na Playlist do curso 5

\subsection{Tópicos Abordados no Curso}

Nos itens seguintes, são apresentados os resumos de todas as aulas ministradas na turma de 2017.

- Apresentação do curso, conceitos gerais envolvendo microcontroladores: Nessa aula, foi apresentada a justificativa do curso, o plano de ensino e o grupo idealizador do projeto, também foi apresentado o conceito de microcontroladores e alguns projetos reais utilizando o chip. Após a apresentação dos microcontroladores, os alunos se apresentaram e comentaram um pouco sobre suas experiencias e habilidades.

- Introdução aos paradigmas de programação em C: Alguns paradigmas de programação em $\mathrm{C}$ foram abordados no curso, como operações com bits e a manipulação dos bits de configuração. Na aula de paradigmas de programação, foi aplicado uma técnica de aprendizado denominada programação em par que é também utilizada na metodologia Extreme Programming [Teles 2004]. Essa técnica consiste na programação conjunta com dois alunos, sendo um deles o piloto (o que produz o código de fato) e o copiloto (o aluno que auxilia o piloto). Utilizando-se essa técnica, percebeu-se a maior participação da turma na aula, além do protagonismo dos alunos na construção do conhecimento. Para a experimentação em programação, foi utilizada a IDE Dev-C++ ${ }^{6}$, como ilustrado na Figura 1.

- Configuração e Programação de Periféricos I/O: Após a exposição da visão geral do PIC16F870 e dos paradigmas de programação em C, foram explorados os pinos digitais de entrada e saída do chip. Foram desenvolvidos projetos de semáforos, com display de 7 seguimentos, botões e teclado matricial.

- Configuração e programação para o display LCD: O último dispositivo de saída de dados apresentado foi o display LCD. Na primeira aula, foi apresentado o documento do display LCD 16x2 e os alunos desenvolveram as funções de configuração do mesmo. Após a construção das funções do display, foi montado o

\footnotetext{
${ }^{4}$ https://obsproject.com/pt-br

${ }^{5}$ https://bit.ly/2rBUY1s

${ }^{6}$ http://orwelldevcpp.blogspot.com.br/
} 


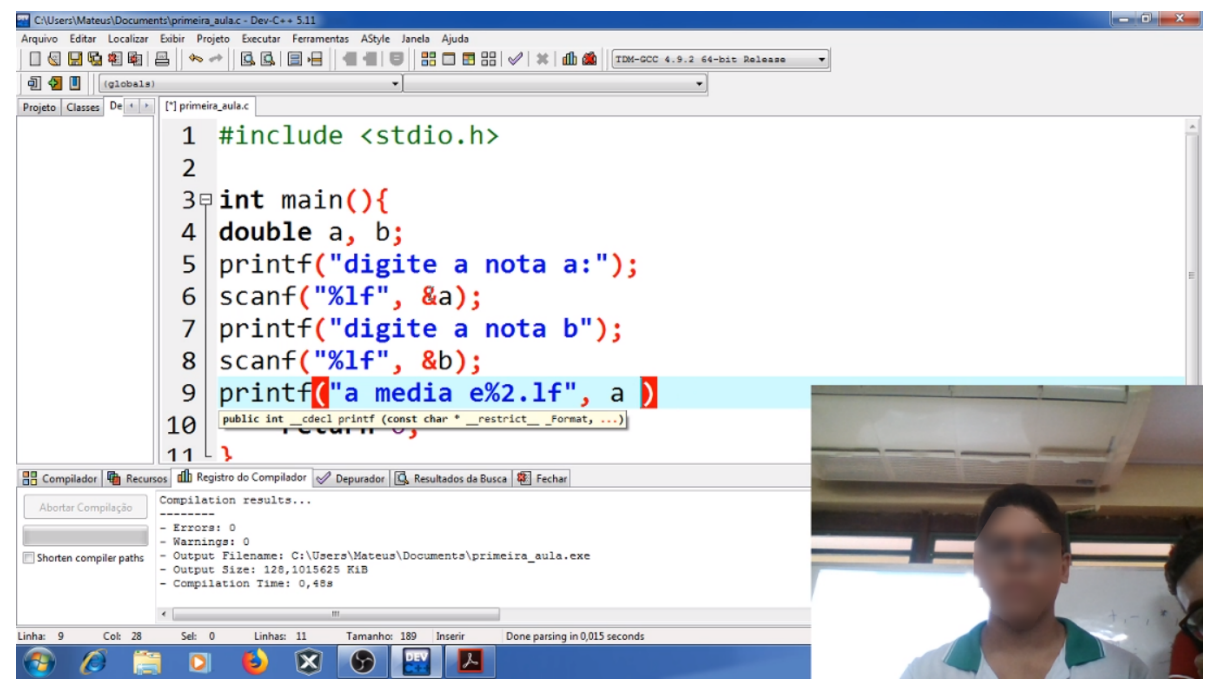

Figura 1. Aplicação da metodologia de programação em par.

circuito do display através de um mapeamento de pinos com o diagrama de pinos do PIC18F4550. Na segunda aula desse tópico, foi realizada uma atividade de construção de uma calculadora, unindo a biblioteca do teclado matricial com a do display, conforme ilustrada na Figura 2.

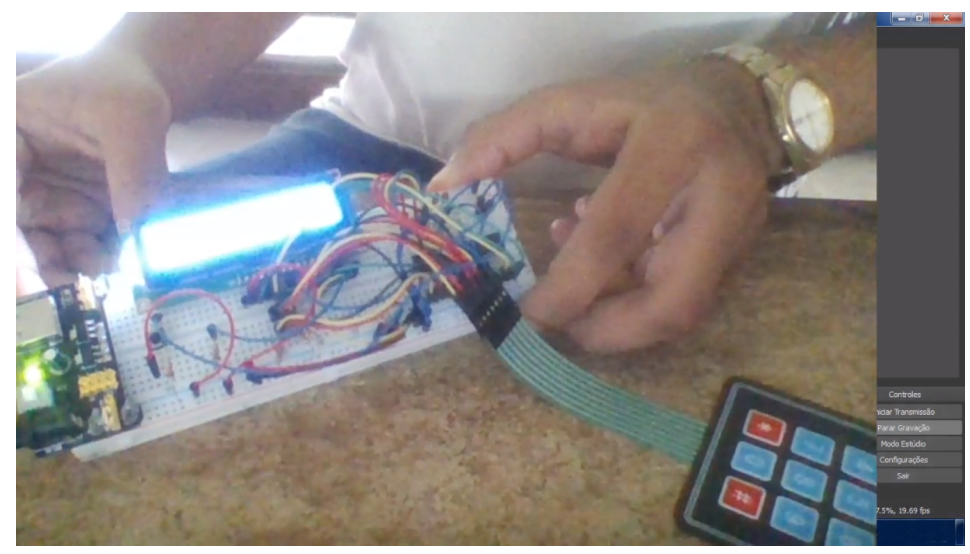

Figura 2. Projetando uma calculadora.

- Introdução a comunicação serial com o módulo USART: Nessa aula, foi explorado o protocolo de comunicação USART, seus modos de operação e suas configurações de operação. Para exemplificar a utilização do protocolo USART, foi utilizado o módulo de comunicação bluetooth, que mais à frente foi utilizado como interface entre um carro de controle remoto e o controle remoto.

- Introdução a leitura de sinais analógicos com o conversor ADC: Neste tópico, foram abordadas as características do módulo ADC e suas configurações básicas. Os alunos utilizaram o sensor de luminosidade LDR para coletar o nível de luminosidade ambiente e enviar esse nível para o PIC em forma de onda analógica. Foi desenvolvido uma aplicação envolvendo a leitura do sensor de luminosidade e o acionamento de uma lâmpada através de um módulo relé.

- Introdução ao controle de servomotores com PWM: Nessa aula, foi abordado 
o módulo Pulse Width Modulation (PWM), bem como suas características e configuração no PIC. Foi utilizado o servomotor $3 \mathrm{~g}$, que é controlado por pulsos periódicos gerados pelo módulo PWM. Esse serviço foi utilizado para controlar o ângulo de direção das rodas de um carro de controle remoto.

- Construção do primeiro robô - $O$ carro de controle remoto: Na parte final do curso, os alunos desenvolveram um carro de controle remoto. Esse projeto final teve como objetivo levar os alunos a uma compreensão maior dos conceitos, e a aplicação do conhecimento adquirido no curso, por meio do desenvolvimento de uma aplicação prática unindo todos conceitos.

\section{Resultados}

Visando identificar se a realização da atividade instigou o interesse dos alunos pela robótica e para obter um feedback acerca do curso, foi solicitado aos alunos que respondessem a um questionário de avaliação. No momento da aplicação do questionário, estavam presentes em sala vinte e nove (29) dos trinta (30) participantes.

No questionário, foram feitas perguntas relacionadas ao conhecimento prévio dos alunos sobre programação, e todos eles já haviam tido contato no curso técnico, como esperado. Poucos alunos $(17,3 \%)$ afirmaram ter um desempenho alto nas disciplinas de programação que fizeram, a maioria $(51,7 \%)$ afirmou ter desempenho mediano e o restante $(31 \%)$ afirmou ter baixo desempenho. Maior parte dos alunos $(65,5 \%)$ manifestou ter interesse por robótica.

No mesmo questionário, foram realizadas perguntas com o intuito de obter um retorno dos alunos sobre o curso. Quase todos os alunos $(93,1 \%)$ consideraram interessante a proposta do curso. Mais da metade dos que responderam ao questionário $(58,6 \%)$ afirmaram que o conteúdo foi aplicado com velocidade ideal e as outras respostas relacionadas à velocidade da aplicação do conteúdo foram distribuídas entre rápida $(27,6 \%)$, muito rápida $(10,3 \%)$ e lenta $(3,4 \%)$.

As últimas perguntas do questionário foram relacionadas à experiência dos alunos ao entrarem em contato com a robótica. Todos os alunos (100\%) passaram a considerar a robótica importante para a sociedade. A maioria dos alunos (69\%) se sentiu estimulado a aprender robótica, enquanto (31\%) não tem interesse.

A resposta dos alunos em relação ao estimulo ao aprendizado de robótica mostrou que a abordagem com microcontroladores não os desestimulou, pelo contrário, esse estilo de ensino de robótica fez os alunos conhecerem de fato como funciona a lógica que rege os dispositivos.

Os resultados obtidos foram satisfatórios, pois os alunos se interessaram pela proposta do curso e se sentiram estimulados a aprenderem mais sobre a área. Essas respostas também mostraram que os alunos acreditam que a robótica é importante para a sociedade, o que foi refletido na abordagem prática da ação.

Todos esses dados podem ser visualizados nos gráficos da Figura 3 .

\section{Conclusão e Trabalhos Futuros}

Este trabalho apresentou um relato de experiência do PET TI - UFC Campus Quixadá no ensino de robótica em uma escola profissional de ensino médio utilizando microcontro- 


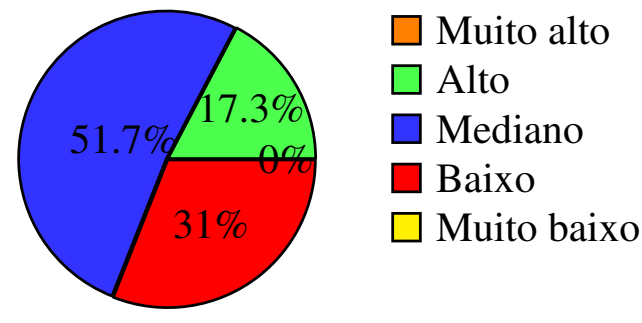

(a) Desempenho dos alunos nas disciplinas de programação vistas no curso técnico.

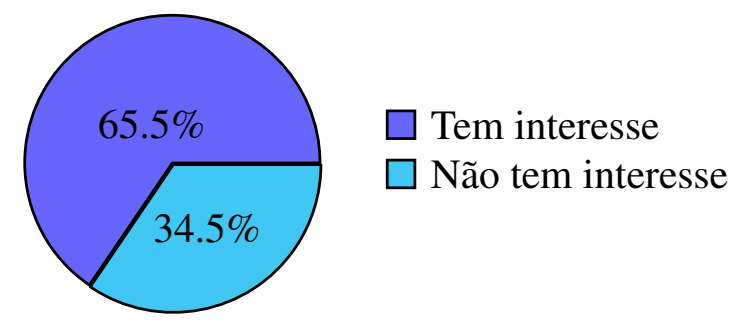

(b) Interesse dos alunos por robótica

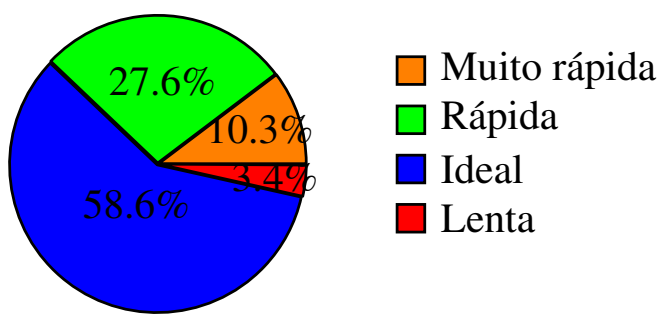

(c) Avaliação dos alunos relacionada a velocidade de aplicação do conteúdo.

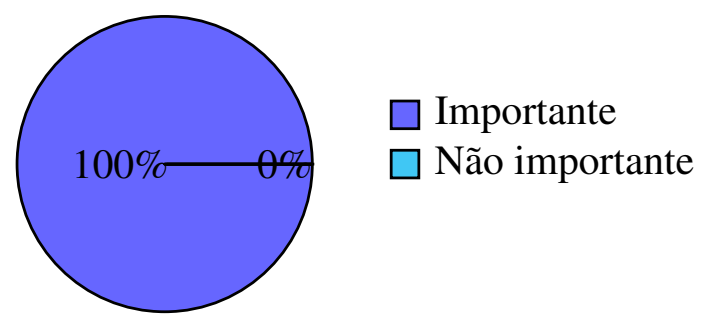

(e) Resposta dos alunos sobre a importância da robótica para a sociedade.

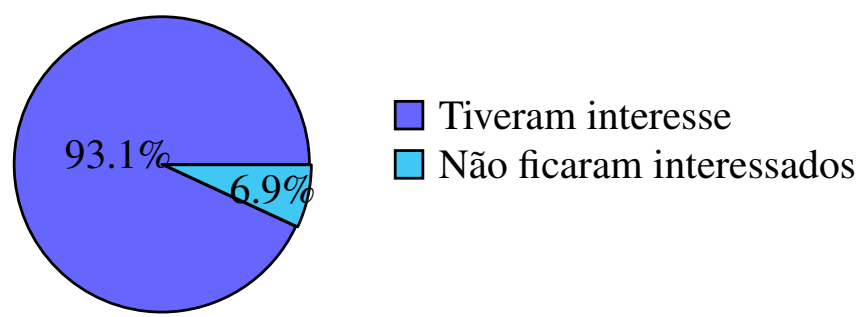

(d) Interesse dos alunos pela proposta do curso.

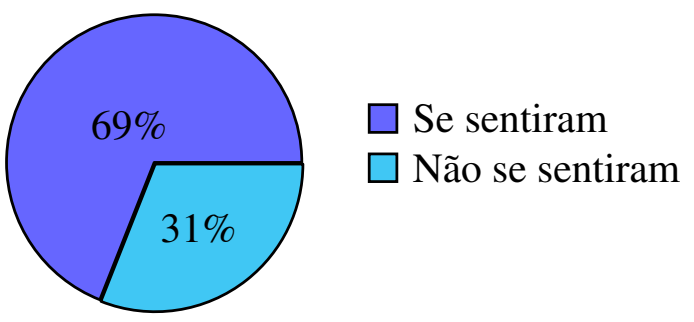

(f) Resposta dos alunos sobre se sentirem estimulados a aprender robótica, ou não.

Figura 3. Gráficos de repostas

ladores. A ação visou o incentivo aos jovens alunos na área de exatas, não só ensinando programação, mas à construção de um carrinho de controle remoto com hardware de baixo custo. A partir das experiências vividas e dos dados coletados, foi possível inferir que os alunos conseguiram compreender os temas abordados no curso e que passaram a ter um interesse maior por aprender sobre robótica.

A experiência de ensino contribuiu também para o aprendizado dos facilitadores, pois estes vivenciaram a experiência ensino aprendizado do ponto de vista docente. $\mathrm{O}$ retorno dos alunos em relação a configurações de periféricos foi notável, pois eles compreenderam o funcionamento do dispositivo e construíram as bibliotecas, mesmo com uma restrita carga horária por tópico. A construção de slides compactos para a introdução dos conteúdos tornou a explicação teórica breve e objetiva, adquirindo com isso mais atenção dos alunos.

Como trabalhos futuros, pretende-se realizar à aplicação de novas metodologias de ensino, como a Aprendizagem Baseada em Problemas (PBL) [Ribeiro 2008] para comparações entre os resultados obtidos, de modo a obter uma melhoria do curso no decorrer 
das próximas ações, além disso também é pretendido realizar um estudo para verificar se os conceitos aprendidos no projeto influenciaram do desempenho dos alunos nas demais disciplinas do curso técnico em Informática.

\section{Referências}

[Avila et al. 2016] Avila, L., Bernardini, F. C., and Moratori, P. (2016). O uso de robótica para aprendizado de programação integrando alunos de educação básica e ensino superior. In XXIV Workshop de Educação em Computação-WEI. Porto Alegre, RS, Brasil.

[Barrientos and Barrientos 2007] Barrientos, A. and Barrientos, A. (2007). Fundamentos de robótica. Technical report, e-libro, Corp.

[Benitti et al. 2009] Benitti, F. B. V., Vahldick, A., Urban, D. L., Krueger, M. L., and Halma, A. (2009). Experimentação com robótica educativa no ensino médio: ambiente, atividades e resultados. In Anais do Workshop de Informática na Escola, volume 1, pages 1811-1820.

[Coutinho et al. 2009] Coutinho, C. P., Sousa, A., Dias, A., Bessa, F., Ferreira, M. J. R. C., and Vieira, S. R. (2009). Investigação-acção: metodologia preferencial nas práticas educativas. Revista Psicologia, Educação e Cultura.

[de Jesus and Cristaldo 2014] de Jesus, L. and Cristaldo, M. F. (2014). Uma abordagem utilizando lego mindstorms education ev3 para verificar o desempenho acadêmico dos estudantes do instituto federal de educação, ciência e tecnologia de mato grosso do sul do câmpus aquidauana. In Brazilian Symposium on Computers in Education (Simpósio Brasileiro de Informática na Educação-SBIE), volume 25, page 1198.

[Martins 2005] Martins, N. A. (2005). Sistemas microcontrolados. Uma abordagem com o Microcontrolador PIC 16F84. Editora Novatec Ltda, $1^{a}$ edição.

[Miyagi and Villani 2008] Miyagi, P. E. and Villani, E. (2008). Mechatronics as a solution for system automation. Journal of Exact Sciences, 5(2).

[Nesi et al. 2014] Nesi, I. C., Nesi Junior, V., et al. (2014). Robótica educacional: uma proposta curricular para o ensino médio.

[Neto et al. 2015] Neto, R. P. B., Santana, A. M., Rocha, D. P., and Souza, A. (2015). Robôtica na educação: Uma revisão sistemática dos últimos 10 anos. In Brazilian Symposium on Computers in Education (Simpósio Brasileiro de Informática na EducaçãoSBIE), volume 26, page 386.

[Ordoñez et al. 2005] Ordoñez, E. D. M., Penteado, C. G., and da Silva, A. C. R. (2005). Microcontroladores e FPGAs: aplicações em automação. Novatec Editora.

[Ribeiro 2008] Ribeiro, L. R. d. C. (2008). Aprendizagem baseada em problemas (pbl): uma experiência no ensino superior.

[Santos et al. 2010] Santos, F. L., Nascimento, F. M. S., and Bezerra, R. M. (2010). Reduc: A robótica educacional como abordagem de baixo custo para o ensino de computação em cursos técnicos e tecnológicos. In Anais do Workshop de Informática na Escola, volume 1, pages 1304-1313.

[Teles 2004] Teles, V. M. (2004). Extreme programming. São Paulo: Novatec. 